\title{
Efeito do tamoxifeno no perfil lipídico de ratos diabéticos por estreptozotocina ${ }^{1}$
}

\author{
Francisco C. Nogueira Junior'2, Daniel A. Coelho², Maria Margareth C.Almeida ${ }^{3}$, Teresa Cristina P. Silva ${ }^{3}$, \\ Elaine Cristina S. Ferreira ${ }^{3}$, Ulisvaldo Bruno O. Macedo ${ }^{4}$, Francisco Paulo F. Neto ${ }^{4}$, \\ José Brandão Neto ${ }^{5}$, Maria das Graças Almeida ${ }^{6}$,Adriana Augusto de Rezende ${ }^{7}$
}

Nogueira-Junior FC, Coelho DA, Almeida MMC, Silva TCP, Ferreira ECS, Macedo UBO, Neto FPF, Brandão-Neto J, Almeida MG, Rezende AA. Efeito do tamoxifeno no perfil lipídico de ratos diabéticos por estreptozotocina. Acta Cir Bras [serial on line] Available from: URL: htt://www.scielo.br/acb.

RESUMO - Objetivo: avaliar o efeito do tamoxifeno no perfil lipídico e renal de ratos controles e diabéticos. Métodos: Foram utilizados 40 ratos fêmeas Wistar (180-220g peso corporal), divididos randomicamente em 4 grupos: $\mathrm{C}(\mathrm{n}=10$, receberam veículo), $\mathrm{T}$ ( $\mathrm{n}=10$, tratados com tamoxifeno, $0,3 \mathrm{mg} / \mathrm{kg} /$ dia), $\mathrm{D}$ ( $\mathrm{n}=10$, diabéticos induzidos por estreptozotocina, $45 \mathrm{mg} / \mathrm{Kg}$ ) e DT ( $\mathrm{n}=10$, diabéticos tratados com tamoxifeno). Foram dosados os analitos, glicose, colesterol total, triglicérides, proteínas totais, albumina, uréia e creatinina utilizando Kits Labtest através do analisador Cobas Mira (Alemanha,1996). Resultados: o grupo T apresentou diminuição do colesterol total e triglicérides em relação ao $\mathrm{C}$, e o grupo $\mathrm{D}$ um aumento em relação aos demais. Para as proteínas totais foi observado um aumento no Grupo T em relação ao C. A albumina diminuiu nos grupos D e DT em relação aos grupos Ce T. Nos níveis de uréia houve um aumento no grupo D e DT em relação aos grupos C e T. Conclusão: Em relação ao perfil lipídico foi constatado que durante o período de 60 dias o tratamento com tamoxifeno promoveu uma diminuição dos níveis séricos de colesterol e triglicérides, mesmo associado a condição de Diabetes mellitus.

DESCRITORES: Diabetes mellitus. Tamoxifeno. Tolesterol total. Triglicérides.

\section{Introdução}

Diabetes mellitus (DM) é um distúrbio metabólico, com etiologias diversas, caracterizado por hiperglicemia e alteração do metabolismo dos carboidratos, lipídios e proteínas, que resulta de uma deficiente secreção de insulina pelas células beta das ilhotas de Langerhans do pâncreas, resistência periférica à ação da insulina, ou ambas .

A hiperglicemia prolongada pode promover o aparecimento de complicações micro e macrovasculares que geralmente surgem depois de 15 anos de doença, embora em alguns pacientes, elas possam estar presentes no início. Um paciente pode apresentar uma ou várias destas complicações. A retinopatia, a neuropatia e a nefropatia são designadas como microvasculares, enquanto que a aterosclerose e suas seqüelas são macrovasculares ${ }^{2}$.

A nefropatia diabética é a causa mais comum de insuficiência renal crônica terminal (IRCT) em pacientes que iniciam tratamento dialítico em países desenvolvidos (30\% dos casos) e em países latino-americano (20\% dos casos). No Brasil, dados das Regiões Sul e Sudeste mostram uma prevalência variável de 9 a 27\%, sendo na Grande São Paulo, a terceira causa de IRCT. A nefropatia diabética é mais comum nos pacientes diabéticos tipo 1 (30 a $40 \%$ dos casos) do que entre os portadores de DM tipo 2 (5 a $20 \%$ dos casos) ${ }^{3}$.

A aterosclerose é um problema freqüente dos pacientes diabéticos, e é mais grave e mais precoce do que na população em geral. A vasculopatia aterosclerótica pode levar a quadros de infarto agudo do miocárdio, que nos diabéticos muitas vezes são silenciosos, e ao acidente vascular cerebral. O diabético pode apresentar, também, quadro de cardiomiopatia com insuficiência cardíaca, mesmo na ausência de doença coronariana .

Diante da necessidade de uma melhor compreensão das complicações associadas ao diabetes, a indução do diabetes em modelos experimentais vêm sendo freqüentemente utilizada, mesmo porque alguns experimentos não poderiam ser realizados em humanos, além de simularem muitos aspectos das complicações do diabetes ${ }^{5}$.

O Tamoxifeno (TAM) é o agente antiestrogênico não esteroidal, comumente utilizado no tratamento do câncer de mama e mais recentemente seu uso tem sido aprovado na quimioprevenção em mulheres com elevado risco de desenvolvimento de câncer de mama. Atua como protetor

1. Trabalho realizado no Programa de Pós-Graduação em Ciências Farmacêuticas da Universidade Federal do Rio Grande do Norte - UFRN, Natal - RN, Brasil.

2. Famacêutico Bioquímico Graduado pela UFRN.

3. Aluna de Mestrado do Programa de Pós-Graduação em Ciências Farmacêuticas da UFRN.

4. Acadêmico de Farmácia da UFRN, bolsista do programa de Iniciação Cientifica PIBIC/ CNPq.

5. Professor, Doutor, Coordenador do Programa de Pós-Graduação do Centro de Ciências da Saúde da UFRN

6. Professor, Doutor, Coordenador do Programa de Pós-Graduação em Ciências Farmacêuticas da UFRN.

7. Professor, Doutor, Vice-coordenador do Programa de Pós-Graduação em Ciências Farmacêuticas da UFRN. 
das doenças coronarianas em humanos, bem como em diferentes modelos de aterosclerose em animais, através do bloqueio da formação da placa de ateroma .

O mecanismo pelo qual o tamoxifeno exerce seu efeito nos lipídios e lipoproteínas não está totalmente esclarecido. Porém estudos sugerem que o tamoxifeno pode atuar como agonista no receptor de estrógeno no fígado, e antagonista em outros tecidos . O efeito agonista do estrógeno no fígado altera o metabolismo e transporte das lipoproteínas tendo assim um importante papel nas alterações do perfil lipídico, desencadeando a redução do colesterol total e LDL, bem como o aumento do colesterol HDL e a hipertrigliceridemia, esta última considerada como complicação do uso de tamoxifeno ${ }^{8}$.

Em relação a hipertrigliceridemia tem sido proposto que pode ser devido um aumento da síntese hepática de VLDL, ou pela redução do catabolismo de VLDL e IDL resultante de uma diminuição da atividade das enzimas lipase lipoprotéica (LPL) e lipase triglicéride hepática

Níveis elevados de triglicérides são observados em pacientes com Diabetes mellitus que fazem uso de tamoxifeno. Pacientes pertencentes ao grupo de risco, antes de iniciar o tratamento devem ser orientados a manter uma dieta pobre em gorduras, além da abstinência ao álcool. Em alguns pacientes se faz necessário o uso de medicamentos para reduzir os níveis elevados de triglicérides ${ }^{8}$.

O presente trabalho tem como objetivo avaliar o efeito do tamoxifeno no perfil lipídico e renal de ratos controles e diabéticos por estreptozotocina, considerando que o tamoxifeno desencadeia alterações lipídicas em relação ao colesterol e triglicérides.

\section{Métodos}

\section{Animais}

Foram utilizados ratos fêmeas da linhagem Wistar, de peso entre $180 \mathrm{~g}$ - $220 \mathrm{~g}$, fornecidos pelo Biotério Central do Centro de Ciências da Saúde da Universidade Federal do Rio Grande do Norte, mantidos em gaiolas com no máximo três animais, com água e ração comercial (Purina) ad libitum. . Durante o período experimental os animais foram mantidos em condições padrões de temperatura e ciclo claro/escuro. Todos os procedimentos foram realizados de acordo com o Guia Padrão de Cuidados e Uso de Animais de Laboratório (1996).

Foram utilizados 40 animais divididos em quatro grupos: Grupo controle (C) 10 animais recebendo apenas veículo (sorbitol + tween $20+$ carboximetilcelulose - CMC); Grupo tamoxifeno $(\mathrm{T})$ - 10 animais com tratamento exclusivo com tamoxifeno; Grupo diabético (D) - 10 animais diabéticos induzidos por estreptozotocina e Grupo diabético com tamoxifeno (DT) - 10 animais diabéticos em tratamento com tamoxifeno.
Durante todo o período de 60 dias foi feita a pesagem semanal dos animais e observação do quadro de poliúria, polidipsia e polifagia para os animais diabéticos.

Indução do diabetes mellitus

Após jejum alimentar, água ad libitum, de 12 horas, ratos Wistar fêmeas de aproximadamente 180-220g receberam via intraperitoneal (i.p.) estreptozotocina (Sigma) dissolvida em tampão citrato de sódio 0,01 M, pH 4,5, na proporção de 45 $\mathrm{mg} / \mathrm{Kg}$ de peso corporal ${ }^{5}$.

\section{Tratamento com tamoxifeno}

Os animais foram tratados diariamente com tamoxifeno, por via oral, em uma proporção de $0,3 \mathrm{mg} / \mathrm{kg} / \mathrm{dia}$, por um período de 60 dias. $O$ tamoxifeno foi preparado em solução aquosa de sorbitol 10\% contendo carboximetilcelulose e Tween 20 .

Obtenção e preparo das amostras de sangue

Após o período de 60 dias os animais foram submetidos a jejum alimentar não hídrico, ad libitum, por cerca de 8-10 horas, sacrificados por deslocamento cervical e submetidos a laparotomia para coleta de cerca de $6 \mathrm{~mL}$ de sangue através da veia porta.

O sangue coletado, em um tubo sem anticoagulante, foi centrifugado utilizando uma centrífuga Sigma 2K15 (Alemanha, 2000) a $1.500 \mathrm{rpm}$, durante 10 minutos. O soro obtido foi utilizado para a dosagem de glicose, colesterol total, triglicérides, proteínas totais, albumina, creatinina e uréia, sendo utilizados para estas dosagens, procedimentos de acordo com a metodologia desenvolvida pela Labtest, utilizando equipamento Cobas Mira da Roche (Alemanha, 1996).

\section{Análise estatística}

Os dados obtidos foram submetidos à análise de variância (ANOVA) para saber se existia diferença significativa entre os grupos estudados durante o período de 60 dias, e em seguida o teste de Tukey foi aplicado para detectar quais as médias apresentavam diferença significativa. $\mathrm{O}$ nível de rejeição da hipótese de nulidade foi fixado em $5 \%$ ( $\mathrm{p} £ 0,05)$. Foi utilizado o programa STATISTICA for Windows versão 5.1 e o programa Excel 2000 MicrosoftÒ para a montagem dos bancos de dados e gráficos.

\section{Resultados}

\section{Caracterização do estado diabético}

A caracterização do estado diabético dos animais em estudo foi realizada através do acompanhamento dos níveis glicêmicos e da evolução do peso corporal de cada animal, além da observação dos quadros de polifagia, polidipsia e poliúria durante o período de 60 dias após a indução (dia zero) e após a instalação do diabetes. 
Os níveis glicêmicos, como pode ser observado na Figura 1, apresentaram-se significativamente aumentados nos grupos dos animais diabéticos e diabéticos tratados com tamoxifeno os quais apresentaram glicemia sempre superior a $250 \mathrm{mg} / \mathrm{dL}$. Já os controles apresentaram glicemia sempre inferior a $130 \mathrm{mg} / \mathrm{dL}$.

Em relação ao peso corporal dos animais, avaliados no mesmo período (Figura 2), observou-se que os animais do grupo dos diabéticos e diabéticos com tamoxifeno apresentaram perda de peso corporal atingindo um peso médio de $195 \mathrm{~g}$ e $186 \mathrm{~g}$, respectivamente, quando comparados aos animais controle e tratados exclusivamente com tamoxifeno que chegaram a um peso médio de $215 \mathrm{~g}$ e $206 \mathrm{~g}$, respectivamente.
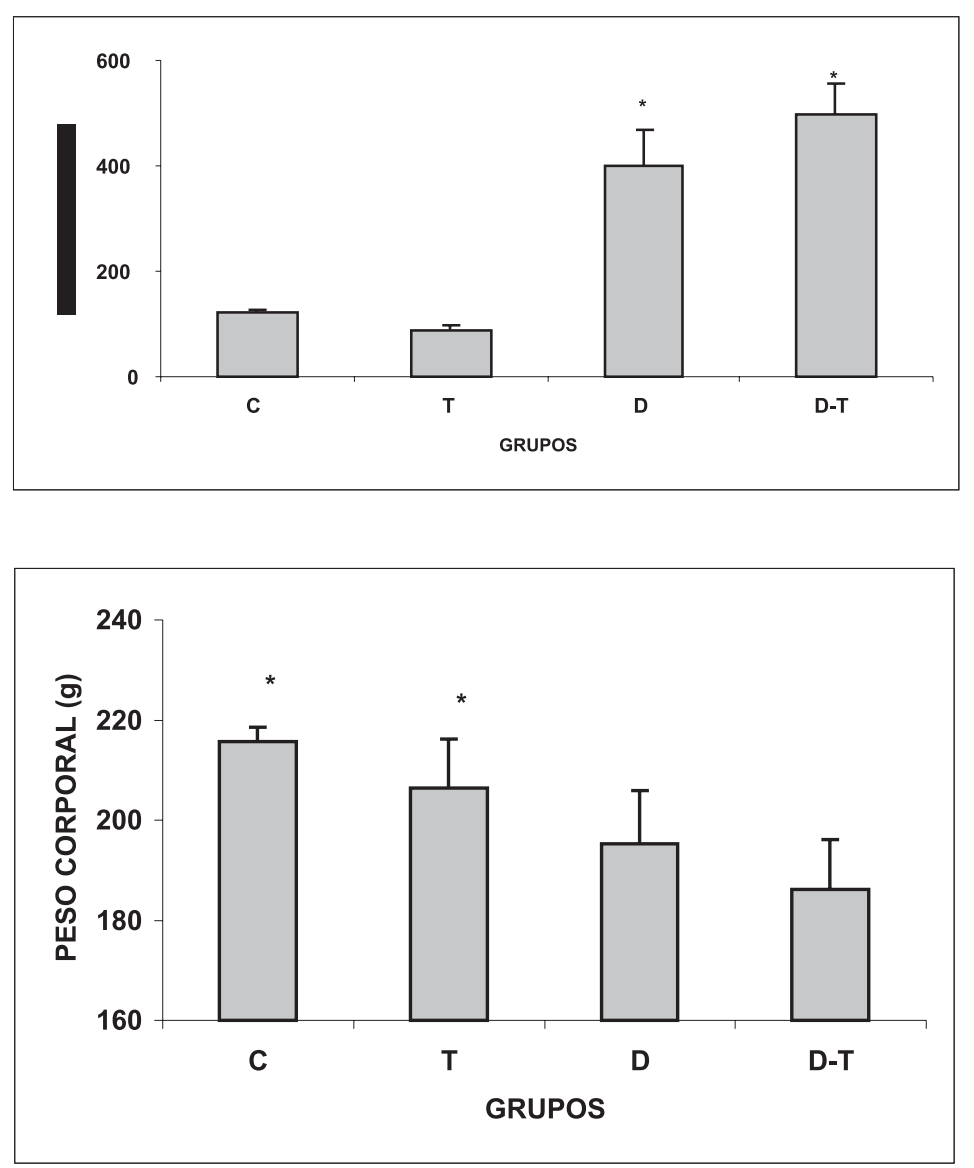

FIGURA 1 - Glicemia dos animais controles (C), animais tratados com tamoxifeno $(\mathrm{T})$, animais diabéticos (D) e animais diabéticos tratados com tamoxifeno (D-T), durante o período de 60 dias. Os resultados são expressos como média \pm desvio padrão.

* $(\mathrm{p}<0,01)$ vs controles

FIGURA 2 - Peso corporal dos animais controles (C), animais tratados com tamoxifeno (T), animais diabéticos (D) e animais diabéticos tratados com tamoxifeno (D-T), durante o período de 60 dias. Os resultados são expressos como média \pm desvio padrão. $*(\mathrm{p}<0,01)$ vs D e D-T, respectivamente

\section{Avaliação de parâmetros bioquímicos}

Os resultados obtidos das concentrações séricas de colesterol e triglicérides acompanhadas durante o período de 60 dias nos gru- pos em estudo estão demonstrados na Figura 3 e 4, respectivamente. Como pode ser observado, os níveis séricos de colesterol e triglicérides apresentaram um aumento significativo para o grupo diabético em relação aos demais grupos.

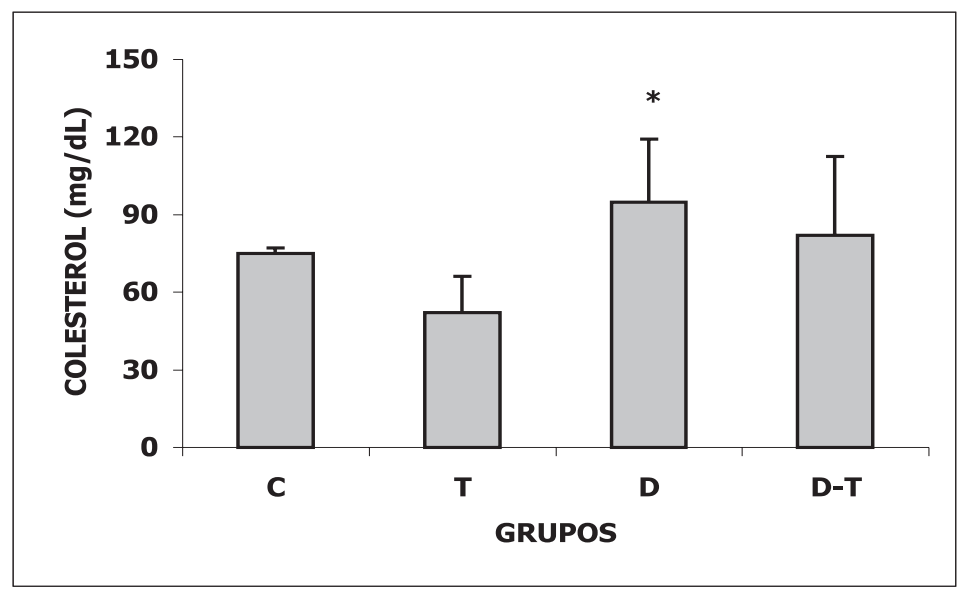

FIGURA 3 - Níveis séricos de colesterol dos animais controles (C), animais tratados com tamoxifeno (T), animais diabéticos (D) e animais diabéticos tratados com tamoxifeno (D-T), durante o período de 60 dias. Os resultados são expressos com média \pm desvio padrão.

$*(\mathrm{p}<0,01)$ vs demais grupos 


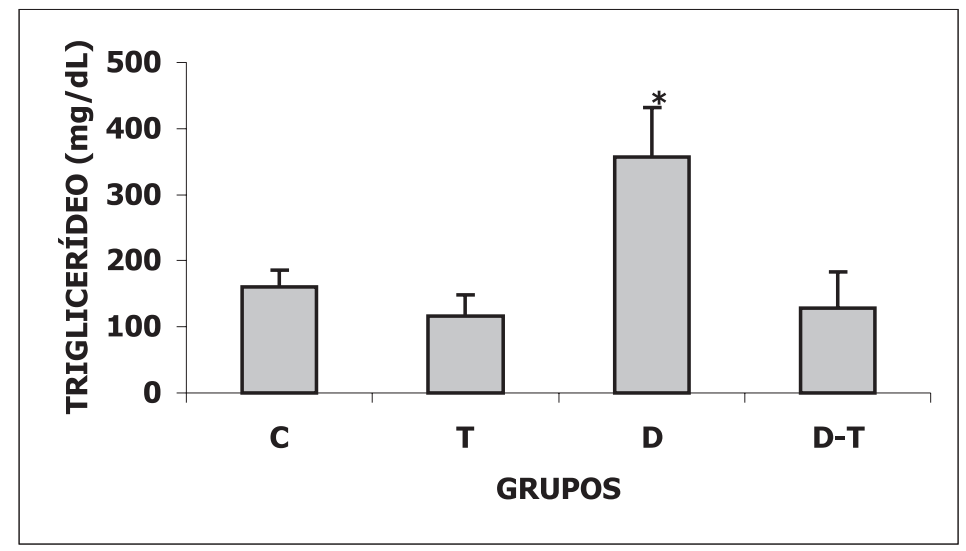

FIGURA 4 - Níveis séricos de triglicérides dos animais controles (C), animais tratados com tamoxifeno ( $\mathrm{T}$ ), animais diabéticos (D) e animais diabéticos tratados com tamoxifeno (D-T), durante o período de 60 dias. Os resultados são expressos com média \pm desvio padrão.

$*(\mathrm{p}<0,01)$ vs demais grupos

A Figura 5 representa a dosagem sérica de Proteínas Totais durante o período de 60 dias. Como pode ser observado, os animais tratados com tamoxifeno apresentaram aumento significativo em relação ao grupo controle.
Em relação aos níveis séricos de albumina durante o período de 60 dias demonstrados na Figura 6, não foi observada diferença entre os grupos controle e tratados com tamoxifeno. Foi notada uma diminuição para os grupos diabéticos e diabéticos tratados em relação aos animais controle e grupo TAM.
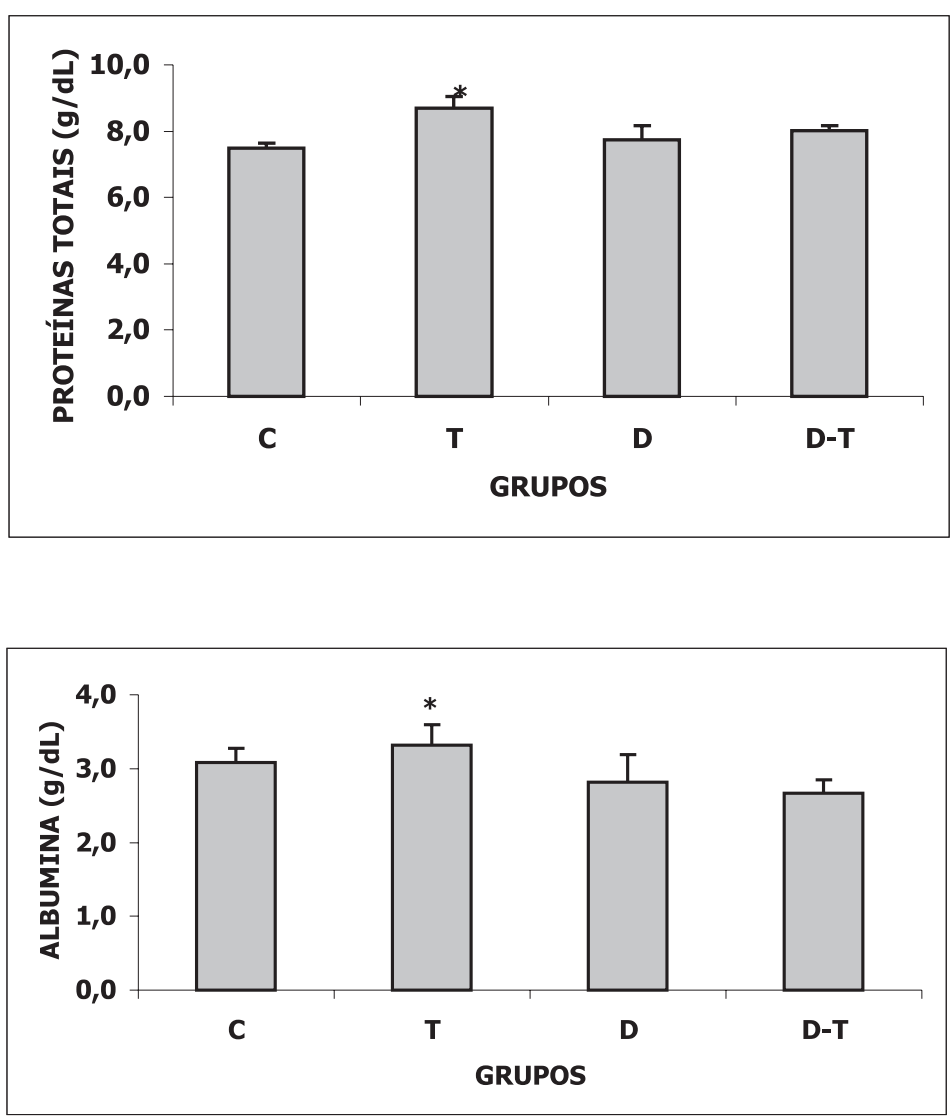

FIGURA 5 - Níveis séricos de Proteínas Totais dos animais controles $(\mathrm{C})$, animais tratados com tamoxifeno (T), animais diabéticos (D) e animais diabéticos tratados com tamoxifeno (D-T), durante o período de 60 dias. Os resultados são expressos com média \pm desvio padrão.

$*(\mathrm{p}<0,01)$ vs $\mathrm{C}$

FIGURA 6 - Níveis séricos de Albumina dos animais controles (C), animais tratados com tamoxifeno $(\mathrm{T})$, animais diabéticos (D) e animais diabéticos tratados com tamoxifeno (D-T), durante o período de 60 dias. Os resultados são expressos com média \pm desvio padrão.

$*(\mathrm{p}<0,01)$ vs D e D-T
A Figura 7 apresenta os valores obtidos para a concentração sérica de creatinina nos grupos estudados, sendo esta considerada um importante parâmetro bioquímico na avaliação metabólica desses animais. Como pode ser observado, não foram determinadas diferenças significativas nos valores da concentração de creatinina entre os grupos, até o período de 60 dias, uma vez que estes resultados estão dentro do intervalo dos valores referência para a creatinina.
A determinação da concentração sérica de uréia, realizada nos grupos em estudo durante o período de 60 dias (Figura 8), revelou que o tratamento com tamoxifeno não promoveu alteração nos níveis de uréia em relação aos animais controle, mas para o grupo diabético e na associação do Diabetes mellitus e tamoxifeno houve uma aumento significativo da concentração sérica de uréia em relação aos grupos controle e tamoxifeno. 

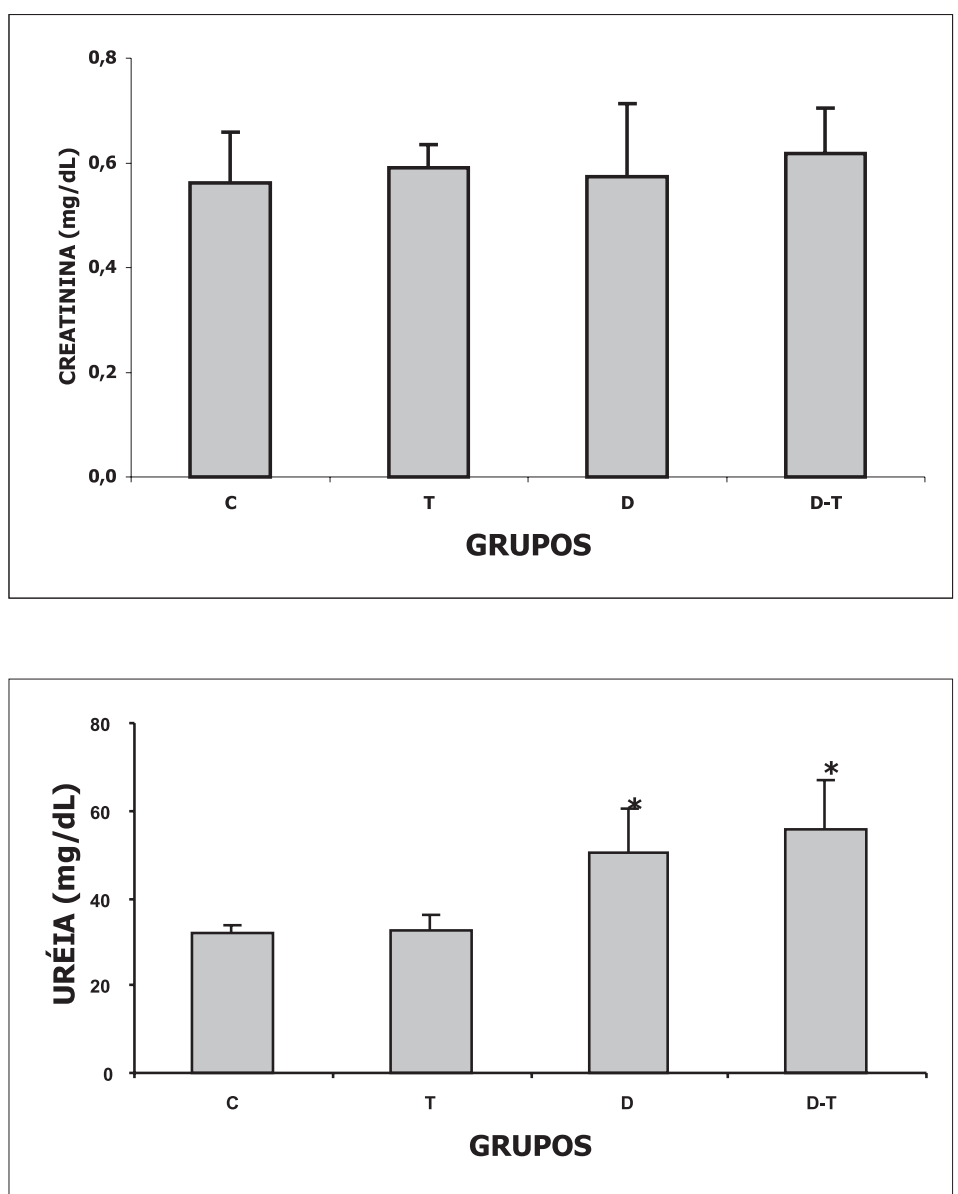

FIGURA 7 - Níveis séricos de Creatinina dos animais controles (C), animais tratados com tamoxifeno (T), animais diabéticos (D) e animais diabéticos tratados com tamoxifeno (D-T), durante o período de 60 dias. Os resultados são expressos com média \pm desvio padrão.

FIGURA 8 - Níveis séricos de uréia dos animais controles (C), animais tratados com tamoxifeno (T), animais diabéticos (D) e animais diabéticos tratados com tamoxifeno (D-T), durante o período de 60 dias. Os resultados são expressos com média \pm desvio padrão. *(p < 0,01) D vs C eT; D-T vs C eT

\section{Discussão}

O diabetes mellitus é uma síndrome crônica, caracterizada pela ausência absoluta ou relativa de insulina e/ou pela resistência à mesma ${ }^{10}$. Constitui um problema de importância crescente em saúde pública. Sua incidência e prevalência estão aumentando, alcançando proporções epidêmicas. Está associado a complicações que comprometem a produtividade, a qualidade de vida e a sobrevida dos indivíduos. Além disso, acarreta altos custos para seu controle metabólico e tratamento de suas complicações ${ }^{11}$.

A aterosclerose é um problema freqüente dos pacientes diabéticos, e é mais grave e mais precoce do que na população em geral. A vasculopatia aterosclerótica pode levar a quadros de infarto agudo do miocárdio, que nos diabéticos muitas vezes são silenciosos ${ }^{4}$. Acredita-se que o tamoxifeno atue como protetor das doenças coronarianas em humanos, bem como em diferentes modelos de aterosclerose em animais, através do bloqueio da formação da placa de ateroma . O mecanismo pelo qual o tamoxifeno exerce seu efeito nos lipídios e lipoproteínas não está totalmente esclarecido. Porém estudos sugerem que o tamoxifeno pode atuar como agonista do estrógeno no fígado alterando o metabolismo e transporte das lipoproteínas tendo assim um importante papel nas alterações do perfil lipídico, desencadeando a redução do colesterol total e LDL, bem como o aumento do colesterol HDL e a hipertrigliceridemia, esta última considerada como complicação do uso de tamoxifeno .
Os resultados obtidos em nosso estudo com os animais pertencentes ao grupo tratado com tamoxifeno apresentaram níveis de colesterol inferiores em relação aos animais controle. Isto sugere a ação do tamoxifeno no sentido de diminuir os níveis de colesterol sérico. Mastroani et al ${ }^{7}$ observaram resultados semelhantes com mulheres (com idade variando de 44 a 70 anos) que fizeram uso de tamoxifeno. De acordo com Gebrim e Lima ${ }^{12}$ o tamoxifeno apresenta ação agonista em tecidos como o hepático o que justificaria a redução do nível sérico do colesterol total em até $15 \%$ devido a diminuição do LDL-colesterol, reduzindo assim as doenças secundárias cardiovasculares.

Para os animais diabéticos foi verificado que durante o período de estudo os níveis séricos de colesterol mostraram-se elevados, devido a própria complicação gerada pela instalação do diabetes. Já o tamoxifeno quando associado ao diabetes, promoveu uma diminuição dos níveis de colesterol se aproximando dos valores encontrados nos animais controles, reforçando a ação do tamoxifeno em reduzir o colesterol sérico.

Considerando que o Diabetes mellitus resulta no aumento da lipólise no tecido adiposo, aumentando os níveis de ácidos graxos sanguíneos haverá uma maior produção de corpos cetônicos pelo fígado. Entretanto, o ácido graxo em excesso captado pelo fígado não será totalmente oxidado a corpos cetônicos pela cetogênese. Assim este excesso de ácido graxo 
será direcionado para a síntese de triglicérides que será transformado em VLDL. Considerando que as VLDLs em excesso não serão depuradas pela lipase lipoprotéica haverá a instalação de uma hipertrigliceridemia. Além disso, a quantidade desta enzima é dependente do nível de insulina no sangue, o que reforça o quadro de hipertrigliceridemia em condição de Diabetes mellitus ${ }^{13}$.

Durante o período de estudo foi observado que os animais tratados com tamoxifeno apresentaram níveis séricos de triglicérides discretamente inferiores aos animais controle, provavelmente devido ao período de estudo ser considerado curto para verificar alguma alteração nos níveis de triglicérides. STONE et al ${ }^{14}$ verificaram que o tratamento com tamoxifeno necessita de períodos superiores a 2 anos para que seja observado alterações nos níveis séricos de triglicérides, uma vez que a hipertrigliceridemia não é visível em períodos curtos.

Os animais que fazem parte do grupo diabético tratados com tamoxifeno apresentaram níveis de triglicérides significativamente inferiores em relação aos diabéticos, indicando uma redução nos triglicérides provavelmente pelo uso de tamoxifeno. Já a literatura relata o contrário, onde níveis elevados de triglicérides são observados em pacientes diabéticos e que fazem uso de tamoxifeno. Taira et al observaram a ação do tamoxifeno, a partir do estudo de uma paciente de 71 anos que possuía um longo histórico de diabetes, e apresentava uma severa hipertrigliceridemia $(2106 \mathrm{mg} / \mathrm{dL})$ provavelmente em virtude do tratamento com tamoxifeno durante o período de 7 anos, embora ela apresente histórico de dislipidemia.

Considerando que o presente trabalho foi realizado em um período de apenas 60 dias, seria precipitado qualquer tipo de conclusão a respeito da ação do tamoxifeno sobre os níveis séricos de triglicérides.

Em relação às proteínas totais o aumento nos animais tratados com TAM poderia ser justificado pela resposta de fase aguda e o aumento das globulinas (frações a a b e g). Este aumento seria desencadeado pela ação do tamóníxifeno, uma vez que proteinogramas realizados no período de 30 dias para animais tratados com tamoxifeno apresentaram um aumento significativo das frações a e a caracterizando uma reposta de fase aguda (resultados não mostrados).

Os animais diabéticos por sua vez apresentaram níveis semelhantes em relação aos animais controle, o que indica até o período estudado que os níveis de proteínas totais não sofreram influência da instalação do diabetes. Entre os grupos dos animais controle, diabéticos e diabéticos tratados com tamoxifeno não foram observadas diferenças significativas. Entretanto, deve ser considerado que há uma tendência de diminuição de proteínas totais do grupo diabético com tamoxifeno em relação ao grupo com tamoxifeno, mas que poderia estar associado ao diabetes, já que para o grupo diabético houve o mesmo comportamento.
Para a albumina, a redução para os grupos tamoxifeno e diabéticos com tamoxifeno poderia estar associada a um possível processo inflamatório, visto que a albumina é uma proteína de fase aguda negativa. Além disso, esta redução poderia estar associada às glicações não-enzimáticas causadas pelo estado de hiperglicemia do Diabetes mellitus, diminuindo assim a concentração de albumina livre e aumentando a concentração de frutosamina ${ }^{16}$. Por outro lado, a diminuição da albumina pode estar associada a uma provável microalbuminúria, geralmente causada pela nefropatia diabética, em virtude da instalação do quadro de Diabetes mellitus ${ }^{3}$.

A redução da concentração sérica de albumina, poderia ainda estar associada a uma lesão hepática e possível comprometimento da síntese protéica, uma vez que a biotransformação do tamoxifeno ocorre a nível hepático e seus metabólitos poderiam causar lesão do DNA, já que são indicados como sendo genotóxico e hepatocarcinogênico para o uso em animais ${ }^{17}$.

Os animais diabéticos e diabéticos com tamoxifeno não apresentaram alterações nos níveis de creatinina em relação aos demais grupos. Para a uréia, os animais do grupo D e DT apresentaram níveis superiores em relação aos demais grupos, em decorrência de uma provável complicação renal desencadeada pelo diabetes, bem como pelo fato da falta de insulina levar a uma exacerbação de processos catabólicos no organismo, envolvendo carboidratos, gorduras e proteínas. Neste sentido, em condição diabética a síntese protéica encontra-se diminuída em todos os tecidos, aumentando a atividade proteolítica muscular com aumento de afluxo de aminoácidos para o fígado e estes aminoácidos elevam os níveis séricos de compostos nitrogenados como a uréia e creatinina $^{18}$.

\section{Conclusão}

O tratamento com tamoxifeno promoveu uma diminuição dos níveis séricos de colesterol e triglicérides, mesmo associado à condição de Diabetes mellitus, sugerindo um efeito favorável da utilização de tamoxifeno em mulheres diabéticas, desde que se considere o risco/benefício do seu uso.

\section{Referências}

1. American Diabetes Association: Standards of medical care in diabetes. Diabetes Care 2004; 27: S15-S35.

2. Committee on The Diagnosis and Classification: Report of The Expert Committee on the Diagnosis and Classification of Diabetes Mellitus. Diabetes Care 2003; 25: 55-120.

3. Lopes, LMV, Pereira M, Vilar L, Morais M. Diagnóstico e Tratamento da Nefropatia Diabética. In: Vilar, L, Castellar E, Moura E, Leal E, Machado AC, Teixeira L, Campos R. Endocrinologia clínica. 2ed. Rio de Janeiro: Medsi; 2001. p. 625 - 36. 
4. Recalde-Sánchez A, Kaski JC. Diabetes mellitus, inflamación y aterosclerosis coronária: perspectiva actual y futura. Rev Esp Cardiol 2001; 54: 751-63.

5. Duarte VMG, Ramos AMO, Rezende LA, Macedo UBO, Neto-Brandão J, Almeida MG, Rezende AA. Osteopenia: a bone disorder associated with diabetes mellitus. J Bone Miner Metab 2005; 23: 58-68.

6. Medina P, Payre BL, Bernad J, Bosseri, Pipy B, Silvente-Poirot, Favre G, Faye JC, Poirot M. Tamoxifen is a potente innibitor of cholesterol esterification and prevents the formation of foam cells. J Pharmcol Exp Ther 2004; 308:1165-73.

7. Mastroanni A, Bellati C.Increased plasma HDL-Cholesterol and apo A-I in breast cancer patients undergoing adjuvant tamoxifen therapy Can Soc Clin Chem 2000; 33:513-6.

8. Milionis HJ, Liberopoulos EN, Elisaf MS. Tamoxifen-induced hypertrigyceridemia in association with diabetes mellitus. Diabetes Metab 2001; 27: 160-3.

9. Hozumi Y, Kawano M, Saito T, Miyata M. Effect of tamoxifen on serum lipid metabolism. J Clin Endocrinol Metab 1998; 8:1633-5.

10. Abuja, P.M.; Albertini, R. Methods for monitoring oxidative stress, lipid peroxidation and oxidation resistence of lipoproteins. Clin Chim Acta 2001; 306: 1-17.
11. Consenso de Diabetes 2002. Diagnóstico e classificação do diabetes melito e tratamento do diabetes melito tipo 2. Sociedade Brasileira de Diabetes. Rio de Janeiro: Diagraphic, 2003.

12. Gebrim LH, Lima GR. Quimioprevenção primária e secundária do carcinoma de mama: In: Prado FC, Ramos J, Valle JR. Atualização Terapêutica 2003. 21ed. São Paulo: Artes Médicas; 2003.

13. Contreras F, Rivera M, Vásquez JF, Yánez CJB, De la Parte MA, Velasco M. Diabetes e Hipertensión Aspectos Clínicos y Terapêuticos. Arch Vzlanos Farmacol Terap 2000;19:9-24.

14. Stone NJ. Secondary causes of hyperlipidemia. Med Clin N Am 1999; 78: 117-41.

15. Taira M, Takasu N, Komiya I. Severe hypertriglyceridemia induced by tamoxifen. Nippon Romen Igakkai Zasshi 1998; 5:858-60.

16. Ahmed N. Advanced glycation endproducts-role in pathology of diabetic complications. Diabetes Res Clin Pract 2005;67:3-21.

17. Kärki A, Mantyla E, Hirsimaki Y, Karlsson S, Toikkanen S, Hirsimaki P. Comparision of the effects of tamoxifen and toremifene on rat hepatocarcinogenesis. Arch Toxicol 2000; 74:249-56.

18. Whelton A, Watson AJ, Rock RC. Metabólitos Nitrotenados e Função Renal. : In: Burtis CA, Ashwood ER. Fundamentos de química clínica. 4ed. Rio de Janeiro: Guanabara Koogan; 1996. p. 553-74.

Nogueira-Junior FC, Coelho DA, Almeida MMC, Silva TCP, Ferreira ECS, Macedo UBO, Neto FPF, Brandão-Neto J, Almeida MG, Rezende AA. Effect of tamoxifen in lipids of diabetic rats induced by streptozotocin. Acta Cir Bras [serial on line] Available from: URL: htt://www.scielo.br/acb.

ABSTRACT - Objective: To evaluate the effect of tamoxifen in the lipidic and renal profile in diabetic and control rats. Methods: 40 female Wistar rats had been used (180-220g corporal weight), divided randomly in 4 groups: $C(n=10$, received vehicle), $T(n=10$, treated with tamoxifen, $0.3 \mathrm{mg} / \mathrm{kg} /$ day), $\mathrm{D}(\mathrm{n}=10$, diabetic induced by streptozotocin, $45 \mathrm{mg} / \mathrm{Kg}$ ) and DT ( $\mathrm{n}=10$, diabetic treated with tamoxifen). Glucose, total cholesterol, triglyceride, total proteins, albumin, urea and creatinin were analyzed using Labtest Kits through the Cobas Mira analyzer (Germany, 1996). Results: Group T presented a reduction of total cholesterol and triglyceride in relation to $\mathrm{C}$, and group $\mathrm{D}$ an increase in relation to the other ones. About the total proteins it was observed an increasing in Group $T$ in relation to $\mathrm{C}$. The albumin diminished in groups $\mathrm{D}$ and $\mathrm{DT}$ in relation to $\mathrm{C}$ and $\mathrm{T}$. The urea levels increased in groups $\mathrm{D}$ and DT in relation to $\mathrm{C}$ and $\mathrm{T}$. Conclusion: In relation to the lipidic profile it was evidenced that during the period of 60 days the treatment with tamoxifen promoted a reduction in cholesterol and triglyceride levels in the serum, even associated to the condition of Diabetes mellitus.

KEYWORDS: Diabetes mellitus. Tamoxifen. Total cholesterol. Triglyceride.

Correspondência:

Adriana Augusto de Rezende

Rua: Pastor Gabino Brelaz 1401, Bloco A, Apto. 101, Capim Macio. CEP 5908210

Telefones: (84) 2079305

(84) 2154377 ou 2154345

e-mail: adrirezende@yahoo.com

Conflito de interesses: nenhum

Fonte de financiamanto: nenhuma 